\title{
THE ARCHITECTURE OF SCIENTIFIC SOFTWARE: THE CONFERENCE
}

In this chapter we provide some background information on the conference that broght together the researchers whose work is described in this volume.

\section{CONFERENCE VENUE}

The conference Software Architectures for Scientific Computing Applications was held in Ottawa, Ontario, Canada on October 2-4, 2000. This was the eight working conference of the International Federation for Information Processing (IFIP) Working Group on Numerical Software (WG2.5) on behalf of the IFIP Technical Committee on Software: Theory and Practice (TC 2). The conference was held on the campus of the National Research Council (NRC) of Canada in Ottawa, where it was hosted by the Institute for Information Technology. The 38 attendees of the focused workshop heard 20 invited presentations. Considerable time was allotted for discussion of issues raised during the talks, and the bulk of this discussion is recorded in this volume.

\section{THE TECHNICAL PROGRAM}

\section{Monday, October 2, 2000}

09:00 Network-based Scientific Computing. Elias Houstis (Purdue University)

09:45 Broadway: Software Architecture for Scientific Computing. Calvin Lin (University of Texas at Austin)

10:30 Break

11:00 Data Management Systems for Scientific Applications. Reagan Moore (San Diego Supercomputer Center)

11:45 Developing Architecture to Support the Implementation and Development of Scientific Computing Applications. Jack J. Dongarra (University of Tennessee)

\section{2:30 Lunch}


14:00 PETSc and Overture: Lessons Learned Developing an Interface Between Components. Kristopher Buschelman (Argonne National Laboratory)

14:45 On the Role of Mathematical Abstractions for Scientific Computing. Krister Åhlander (University of Bergen)

15:30 Break

16:00 The Virtual Testing Facility Application Framework. J. T. C. Pool (California Institute of Technology)

\section{Tuesday, October 3, 2000}

09:00 Formal Methods for High-Performance Linear Algebra Libraries. Robert van de Geijn (University of Texas at Austin)

09:45 Software Components for Application Development. Anne Trefethen (NAG)

10:30 Break

11:00 Component Technology for High-Performance Scientific Simulation Software. Scott Kohn (Lawrence Livermore National Laboratory)

11:45 A New Approach to Software Integration Frameworks for Multi-Physics Simulation Codes. Milind Bhandarkar (University of Illinois)

12:30 Lunch

14:00 Hierarchical Representation and Computation of Approximate Solutions in Scientific Simulations. Wayne Enright (University of Toronto)

14:45 Software Architecture for the Investigation of Controllable Models with COmplex Data Sets. Dmitry V. Belyshev (Program Systems Institute, Russian Academy of Sciences)

15:30 Break

16:00 Multi-Language Programming Methodology for HighPerformance Java. Vladimir Getov (University of Westminster and Los Alamos National Laboratory

\section{Wednesday, October 4, 2000}

09:00 A Comprehensive DFT API for Scientific Computing. Ping Tak Peter Tang (Intel Corporation)

09:45 New Generalized Data Structure for Matrices Leading to a Variety of High-Performance Algorithms. Fred Gustavson (IBM)

10:30 Break 
11:00 A Collaborative Code Development Environment for Computational Electromagnetics. David W. Walker (Oak Ridge National Laboratory)

11:45 Code Coupling Using Parallel CORBA Objects. Christophe René (IRISA/IFSIC)

12:30 Lunch

14:00 Object-oriented Modeling of Parallel PDE Solvers. Michael Thuné (Uppsala University)

14:45 A Fortran Interface for POSIX Threads. Richard Hanson (Rice University)

\section{ORGANIZATION}

The Program Committee consisted of Ronald Boisvert, Wayne Enright, Stuart Feldman, Brian Ford, Patrick Gaffney, Morven Gentleman (co-chair), Ian Gladwell, Eric Grosse, Pieter Hemker, Richard Kazman, Mo Mu, James C. T. Pool (co-chair), John Rice, Mary Shaw, Brian Smith, Peter Tang, and Mladen Vouk.

Morven Gentleman served as local arrangements chair. Roger Impey hosted the conference for the NRC, and Nicole Sarault and Guylaine Caron assisted.

The conference was supported with grants from IBM Canada Ltd., the Hewlett-Packard Company, Intel Corporation, and the National Research Council of Canada.

\section{PARTICIPANTS}

Krister Åhlander Dmitry V. Belyshev Milind Bhandarkar

Ronald F. Boisvert

Kristopher Buschelman Bruce Char Jack Dongarra

Bo Einarsson

Wayne Enright Richard Fateman

Alain Gaboriaud Morven Gentleman

Vladimir Getov
University of Bergen, Norway

Russian Academy of Sciences, Russia

University of Illinois at Urbana-Champaign, USA

National Inst. of Standards and Technology, USA

Argonne National Laboratory, USA

Drexel University, USA

University of Tennessee, USA

Linkoping University, Sweden

University of Toronto, Canada

University of California at Berkeley, USA

Centre National d'Etudes Spatiales, France

Dalhousie University, Canada

Los Alamos National Laboratory, USA 
Ian Gladwell

Fred G. Gustavson

Richard J Hanson

Elias N. Houstis

Roger Impey

Peter Klein

Scott Kohn

Calvin Lin

Reagan Moore

Ivor Philips

Laurent Plantié

James C. T. Pool

Thierry Priol

Christophe René

John R. Rice

Dennis Salahub

Masaaki Shimasaki

Brian T. Smith

Ping Tak Peter Tang

Michael Thuné

Anne Trefether

Robert A. van de Geijn

Mladen Vouk

David Walker

Margaret H. Wright
Southern Methodist University, USA

IBM Corporation, USA

Rice University, USA

Purdue University, USA

National Research Council, Canada

Institute for Industrial Mathematics, Germany

Lawrence Livermore National Laboratory, USA

University of Texas at Austin, USA

San Diego Supercomputer Center, USA

Boeing, USA

CERFACS, France

California Institute of Technology, USA

IRISA/INRIA, France

IRISA/IFSIC, France

Purdue University, USA

Nationa Research Council, Canada

Kyoto University, Japan

University of New Mexico, USA

Intel Corporation, USA

Uppsala University, Sweden

NAG Ltd., United Kingdom

University of Texas at Austin, USA

North Carolina State University, USA

Oak Ridge National Laboratory, USA

Bell Laboratories, USA

\section{IFIP WORKING GROUP 2.5}

In 1974 IFIP established the Working Group on Numerical Software under the auspices of its Technical Committee on Software: Theory and Practice. The group started with 13 members and has since grown to a membership of more than 30 .

The aim of Working Group 2.5 is to improve the quality of numerical computation by promoting the development and availability of sound numerical software. Objectives within the scope of the Working Group 2.5 are as follows.

- The definition from a numerical standpoint of a set of hardware and software features for a computing system.

- The development and improvement of programming languages for numerical computation. 
- The establishment of guidelines for comparison of subroutines from different numerical program libraries.

- The establishment of guidelines for documentation, testing, distribution and maintenance of numerical program libraries.

- The exchange of information concerning numerical software and determination of the needs of computer users.

The following mode of work has been established within WG 2.5.

1 Working group meetings

The group meets roughly once a year. (There have been 27 meetings so far). At these meetings, areas of activity and the proper means of achieving results are defined. Reports on current or completed activities are given and discussed.

2 Projects

Most activities take the form of projects. One or several members of the group assume the responsibility to pursue a given subject matter in collaboration with other scientists in the field. Results of projects are either published through standard channels or, in special cases, may take the form of an IFIP-document.

3 Working Conferences

This is a standard means of IFIP-activity: $30-70$ experts are invited to meet to discuss and advance a narrow technical subject area. The proceedings of a working conference appear as a book.

For further information, see http://www.nsc.liu.se/wg25.html . 